\title{
Compression of self-ion implanted Iron Micropillars.
}

\author{
E.M. Grieveson*, D.E.J. Armstrong*, Sho Xu*, S.G. Roberts* \\ *University of Oxford, Parks Road, Oxford, OX1 3PH, UK, +44(1865)273768
}

\section{Highlights}

- Self-ion implantation used to cause cascade damage in pure iron

- Increase in hardness measured in implanted region using nanoindentation

- Micropillars manufactured and tested in both implanted and unimplanted material

- Marked difference in deformation mechanisms in each set of pillars seen using scanning electron microscopy

- No difference in yield stress seen, suggesting it is difficult to use micro-compression to understand bulk properties

\begin{abstract}
Ion implantation causes displacement damage in materials and can cause changes to the mechanical properties due to the formation of dislocation loops. Samples of pure Fe were subjected to $\mathrm{Fe}^{+}$implantation at $275^{\circ} \mathrm{C}$, producing damage of $\sim 6 \mathrm{dpa}$ to $\sim 1 \mu \mathrm{m}$ depth.

Micropillars were manufactured in cross-section specimens of implanted and bulk material and compressed using a nanoindenter. Nanoindentation into implanted as compared to unimplanted material shows an increase in hardness. However the implanted pillars have a deformation mode which differs markedly from the unimplanted pillars but there is no change in yieldstress. Suggesting that the controlling mechanism for deformation is different between nanoindentation and micropillar compression and that care is needed if using micropillar compression to extract bulk properties of irradiated materials.
\end{abstract}

\section{Keywords}

nanoindentation; compression test; iron; ion implantation; slip 


\section{Introduction}

Materials proposed for the first wall \& blanket of a fusion power plant such as DEMO include low activation steels based on $\mathrm{Fe}-\mathrm{Cr}$ [1]. Neutron irradiation causes the formation of point defects and dislocation loops [2] and changes the mechanical properties of the materials leading to hardening and embrittlement [3]. Working directly with neutron irradiated materials is time consuming and requires special facilities and so ion-implantation is widely used as a simulation of neutron damage. Typically ion implantation experiments produce damage in the very top layer of the materials (typically $\sim 1-10 \mu \mathrm{m}$ depending on ion species and energies used). This thin layer of damage means that large scale mechanical testing is not possible; however nanoindentation can provide basic data on hardness changes $[\mathbf{4 , 5}, \mathbf{6 , 7 , 8}]$, although uncertainties about the effects of the unimplanted substrate can make data interpretation difficult $[9,10]$. For more detailed information about the properties of the material and to produce a true stress-strain curve which can be easily compared with bulk data, the use of more refined micro-mechanical methods which sample only the ion-implanted layer are needed.

Micromechanical testing methods have been developed in various geometries for bending, compression and tensile testing $[\mathbf{1 1}, \mathbf{1 2}, \mathbf{1 3}, \mathbf{1 4}]$. A simple, and now widely used, test geometry is a compression pillar $[\mathbf{1 5}, \mathbf{1 6}, \mathbf{1 7}$, which gives the advantage of a simple stress state [18] compared to the more complex stress states found in bending experiments. However, research on pillar compression at the micron scale has shown very strong size effects on the yield stresses (smaller pillars being stronger) [19] and on the shape of the stress-strain curves (smaller pillars giving step-like, stochastic, behaviour). This has been attributed to both dislocation starvation [20] and the statistics of a limited number of small dislocation sources [21]. This has limited the applications of such tests to understanding the underlying physical processes that govern the dislocation motion in the pillars while little work has focused on using them to try and understand the properties of bulk engineering alloys.

Several researchers have used micromechanical testing techniques to test the mechanical properties of ion implanted samples. Halliday et al. [5] found an increase of $\approx 500 \mathrm{MPa}$ in yield stress for micro-cantilevers machined in pure iron implanted to 0.54dpa as compared to unimplanted cantilevers. This method has the disadvantage of having to manufacture very thin micro-cantilevers which have an uncertain stress state due to variations in cantilever shape and which are difficult to test in a conventional nanoindenter because of their very low compliance. Pouchon et al.[22] Used both nanoindentation and micropillar compression to investigate the mechanical properties of PM2000 oxide dispersion strengthened steel before and after irradiation with $\mathrm{He}$ ions at $24 \mathrm{MeV}$. The nanoindentation showed significant hardening after irradiation of $\approx 0.3 \mathrm{GPa}$. Micro-compression tests on micropillars produced in the unimplanted material showed similar yield stresses to bulk samples with the deformation seen to occur in a single slip band, however due to difficulties with interpretation of the micropillars in implanted samples those results were not reported. Hosemann et al.[23] Used square micropillar specimens with side lengths of $8 \mu \mathrm{m}$ to investigate changes in mechanical properties of ferritic/martenstic steel irradiated in the Spallation Target Irradiation Program to damage levels from $0-18 \mathrm{dpa}$. They saw an increase in yield stress of $150 \mathrm{MPa}$ at 10dpa, with the general trends in good agreement with macroscopic tests, although absolute values of yield stress were higher as measured by micro-compression. Kiener et al. ref manufactured pillars of diameter $80 \mathrm{~nm}$ to $1500 \mathrm{~nm}$ in proton irradiated copper $(0.8 \mathrm{dpa})$ which were then compressed inside a TEM. They observed an increase of $\approx 100 \mathrm{MPa}-250 \mathrm{MPa}$ in yield stress for irradiated vs non- 
irradiated samples above 400nm diameter. Such in-situ work allows the deformation mechanisms to be observed directly but only allows small pillars to be observed; also it is inherently slow, making it time-consuming to carry out many tests.

\section{Materials and Methods}

Pure iron supplied by Metals, Crystals and Oxides, (Cambridge, UK) was annealed at $500^{\circ} \mathrm{C}$ to produce a grain size of $\sim 100 \mu \mathrm{m}$ diameter, large enough to allow multiple pillars to be milled within single grains. Samples were ground on silicon carbide paper, followed by polishing with $3 \mu \mathrm{m}$ and $1 \mu \mathrm{m}$ diamond pastes and finally using a 40nm colloidal silica $-10 \%$-hydrogenperoxide solution on a chemo-mechanical polishing cloth. Similar polishing by Armstrong et al. [6] has been shown to give a high quality surface finish, free from damage when examined using electron backscattered diffraction and with similar nanoindentation response to electropolished material. The ion implantation of the samples was carried out at the Surrey Ion Beam Centre, UK, using sequential Fe ion doses of $3 \times 10^{15} \mathrm{~cm}^{-2}$ at $2.0 \mathrm{MeV}$ and $1.5 \times 10^{15} \mathrm{~cm}^{-2}$ at $0.5 \mathrm{MeV}$ at $275^{\circ} \mathrm{C}$. The implantation time was 124 minutes for the $2.0 \mathrm{MeV}$ ions and 75 minutes fr the $0.5 \mathrm{MeV}$ ions. The bulk samples were mounted on stainless steel holders in a similar manner to that described in Armstrong et al.[6] and Busby et al.[24] and the temperature monitored by a thermocouple mounted alongside the samples, this gave a reading of $275^{\circ} \mathrm{C}+-10^{\circ} \mathrm{C}$ throughout the experiment. Electropolished $3 \mathrm{~mm}$ discs of the same material were also mounted in a special holder and implanted at the same time to allow TEM studies of the damage structure in material with an identical thermal cycle. The predicted damage profile (using SRIM with an Fe displacement energy of 40eV) is shown in Bushby et al. [24] with two overlapping peaks due to the two beam energies used; this gives a fairly uniform damage profile with an average dose of $6 \mathrm{dpa}$ over the first $0.75 \mu \mathrm{m}$ depth, decreasing to zero at $1 \mu \mathrm{m}$ depth.

After implantation the samples were nickel plated using electroless plating for edge retention during cross-section polishing [25]. A solution of $18 \mathrm{~g}$ nickel chloride, $4.4 \mathrm{~g}$ sodium hydroxide, $40 \mathrm{~g}$ sodium citrate, $20 \mathrm{~g}$ ammonium chloride and $400 \mathrm{ml}$ distilled water was heated to between 90 and $100^{\circ} \mathrm{C}$ and samples were immersed in the boiling solution for 25 minutes producing a Ni coating of $\sim 6 \mu \mathrm{m}$ thickness. The samples were then glued into 'sandwiches' ( Figure 1a). The polished faces of two samples were coated with epoxy resin, pressed together, clamped and left to dry. The sandwiches were cold resin mounted in Kemet epoxy resin VLT in a PTFE mould and cured under vacuum conditions for approximately twelve hours. The mounted sandwiches were then polished as described above, to produce a polished cross section of the ion implanted region, Figure 1a.

\section{Experimental Methods}

A line of Berkovich nanoindents (using a MTS Nano XP with continuous stiffness measurement) was made at a shallow angle to the Ni plating and glue boundary lines close to the area used for pillar testing. Indents (maximum depth 50nm made at a strain rate of 0.05), were spaced $5 \mu \mathrm{m}$ parallel to and $0.1 \mu \mathrm{m}$ perpendicular to the glue line. Figure $1 \mathrm{~b}$ shows an average hardness of $3.1 \mathrm{GPa} \pm 0.6 \mathrm{GPa}$ in the unimplanted region and an average hardness of $5.5 \mathrm{GPa} \pm 0.9 \mathrm{GPa}$ in the implanted region. The horizontal error bars take into account the plastic zone size of the indents. 
The $\mathrm{Fe}^{+}$ion implanted layer is $\sim 1 \mu \mathrm{m}$ deep so the pillars for mechanical testing need to be $<1 \mu \mathrm{m}$ in diameter to ensure the mechanical properties being tested are of the implanted material. In order to produce pillars with close to parallel sides they were annular-milled using $\mathrm{Ga}^{+}$ions in an FEI 200 FIB. A three-stage milling process using a decreasing current each time was used (1nA followed by 300pA followed by 50pA). The finished pillars had diameters slightly less than $1 \mu \mathrm{m}$, and a height of $\approx 8 \mu \mathrm{m}$. Figure 2 shows a tested pillar manufactured on the edge of the sample with the nickel coating and the epoxy resin visible to the left of the pillar.

The micropillars were compression tested using a flat punch with a diameter of $4.5 \mu \mathrm{m}$ to a displacement of 500nm at a loading rate of $5 \mathrm{nms}^{-1}$, using an MTS Nano Indeter XP. The shape and alignment of the punch was calibrated by AFM study of a set of indentations made with it into soft copper. The indentations had flat bases at an angle of $\sim 0.6^{\circ}$ to the horizontal. This tilt error is of a similar order to that introduced during loading the samples into the indentation equipment.

TEM was carried out using a Philips CM20 at 200kV on back thinned $3 \mathrm{~mm}$ discs using electropolishing to allow examination of the produced defects. (more details needed?)

\section{Results and Discussion}

Figure 3 shows engineering stress-strain curves calculated from the load-displacement data recorded during compression. These were calculated using the using the measured dimensions of each pillar. The heights of the pillars were measured using images taken at $10^{\circ}$ tilt angle as this allowed the whole pillar to be imaged, which allowed a strain to be calculated based on the compression of each pillar to 500nm. However it is clear from figure 4 that the deformation of each pillar (especially the unimplanted ones) is very localised and as such a macroscopic strain may have limited physical meaning. Due to the manufacture of the pillars using an annular milling method there is a small taper on each pillar which will result in a variation of stress along the length of the pillar, As seen in figure 4 the observed deformation is always close to the top of the pillar, as such the diameter of the top of each pillar was used to calculate the resultant stress. Since yield points were hard to determine, the flow stress at $0.5 \%$ strain was used compare the various tests; data are shown in Table 1 . The average value for implanted pillars is $260 \pm 125 \mathrm{MPa}$, and for unimplanted pillars is $208 \pm 121 \mathrm{MPa}$; the difference is within experimental error. Electron back scattered diffraction (EBSD) was used to measure the compressive axis of each set of micropillars; the results are also shown in Table 1. For each pillar the Schmid Factor was calculated, assuming slip could occur with $\langle 111\rangle$ slip directions on $\{110\},\{112\}$ or $\{123\}$ planes. In each case this was between 0.481 and 0.499 , with the maximum Schmid factor on a $\{112\}$ or $\{123\}$ plane. Assuming the critical resolved shear stress for slip does not vary strongly with slip system [26], pillar orientation should not have had a strong influence on the test results.

Figure 5 is a TEM image taken in the 111 zone with $\mathrm{g}=110$ showing the damage structure produced after ion implantation. The interstitial type loops have an average diameter of $11 \mathrm{~nm}$ and a density of $4.5 \times 10^{21} \mathrm{~m}^{3}$. 
The compressive yield stresses in the implanted and unimplanted materials are similar, which is different from the nanohardness results which show a clear hardening effect from implantation in the damaged depth, as is commonly found [24]. They also show a similar large range of yield stresses, similar to those seen in other micro compression experiments on pillars of similar diameter, such as Dimiduk et al. [19] who saw a range of $125 \mathrm{MPa}$ in $1 \mu \mathrm{m}$ Ni pillars. Using the well known relation that hardness $\approx 3 x$ flow stress [27] the flow stress of unimplanted pure iron would be predicted from the indentation data to be $\approx 1 \mathrm{GPa}$ and that of the implanted material $\approx 1.8 \mathrm{GPa}$. In both cases these are rather higher than the yield stresses from the pillar experiments, which will be at least partly accounted for by the small size $(50 \mathrm{~nm})$ of the indents, due to the well-established "indentation size effect" [28].

In all pillar specimens, flow occurs in discrete 'strain bursts' seen as horizontal lines on the stress/strain curves. Table 1 shows the number of strain bursts in each sample type up to a strain of 3\%; the number of bursts in the implanted pillars is approximately twice that of the unimplanted pillars, and the size of each strain burst is correspondingly smaller. SEM imaging of the pillars before and after compression (Figure 3) shows a distinct difference in the deformation modes between implanted and unimplanted materials. The unimplanted pillars shear along a small number of narrow widely spaced slip lines, whereas in the implanted pillars all the slip is concentrated in a single wide dense band.

The unimplanted pillars show plastic deformation along a few discrete slip lines, similar to deformation seen in other pillar compression experiments $[29,30]$. This mode of slip is probably due to the small number of available dislocation sources within the pillars [20]; with a typical dislocation density of $1 \times 10^{14} \mathrm{~m}^{-2}$ in a well annealed metal, it would be expected that each pillar would only contain at most a few dislocation sources. The size of the slip steps visible on the surfaces of the micropillars (several hundred nanometres) indicates that several hundred dislocations have propagated from each active dislocation source. Work by Kim et al. [31] has shown that the deformation of pillars of four different bcc metals, (Nb, W, Mo, Ta) have very different morphologies, which was attributed to the differences in the mobilities of screw and edge dislocations in each case. The unimplanted iron pillars in this study have a deformation structure most similar to those found by Kim in niobium, with localised slip. This may correspond to the critical temperature at which it is equally easy to propagate edge [32] or screw [33] dislocations being similar $\left(\mathrm{T}_{\mathrm{c}},=350 \mathrm{~K}\right)$ in the two materials. As suggested by Schnieder et al. [34] it seems likely that once activated the slip occurs on a single slip plane before the dislocations responsible leave the free surface, and sufficient stress must build up to nucleate another dislocation source. Most molecular dynamic and dislocation dynamic modelling of bcc micropillars has shown concentrated on molybdenum and has indicated that dislocations can undergo complex reactions leading to size effects $[\mathbf{3 5}, \mathbf{3 6}]$. However recent MD modelling of iron micropillars has shown the formation of localised slip bands very similar to those seen here in the unimplanted pillars [37].

The stress vs. strain graphs show strain-bursts in the deformation of all pillars. Each burst is due to rapid displacement during loading, corresponding to plastic flow along the visible slip lines. Each strain burst corresponds to a dislocation source (or couple of sources) activating and allowing plastic deformation before a jump in stress to the next strain burst. In the unimplanted material (lower dislocation density), an activated source will run until no more deformation is possible before another source activates (at a higher stress). For all compositions the implanted material shows many more, and shorter, strain bursts than the unimplanted material. There are three possible mechanisms for the changes in yield nature and observed deformation between 
unimplanted and implanted micropillars they are; cascade damage acting as dislocation sources, cross-slip of primary dislocations or the formation of defect free channels. These will be discussed in turn,

Discrete dislocation modelling by Novokshanov et al. [38] has shown while interstitial dislocation loops in iron can act as dislocation sources, this is at stresses in the 2-10 GPa range, which is significantly higher than the yield stresses seen in the micro-compression experiments. Moreover if this was the mechanism which was dominating the deformation in the micropillars then the range of yield stresses seen in the implanted pillars would be expected to be much narrower than that seen in the unimplanated case which is not the case. Thus the initial yield stress of the implanted pillars must still be controlled by prexisiting dislocations in the material. Thus the loops must act as obstacles to the glissile dislocations. The stress concentration this causes then acts to activate a dislocation source on an adjacent slip plane which along with cross slip events will tend to lead to broadening of the first slip band. The frequent triggering of these sources will give rise to the larger number of small strain bursts observed as compared with the unimaplanted micropillars. Alternatively the deformation band could be broadened by the formation of defect free channels. These have been seen experimentally in both neutron $]^{39}$ ] and proton ${ }^{40}$ irradiated iron samples. Recent modelling work by Marian et al. ${ }^{\mathbf{4 1}}$ has shown that irritated iron samples uniaxial stressed show the formation of defect free channels start to from above a critical defect density between $3.6 \times 10^{21} / \mathrm{m}^{3}$ and $8.15 \times 10^{21} / \mathrm{m}^{3}$ which is similar to the defect volume density seen here of $4.5 \times 10^{21} / \mathrm{m}^{3}$.

The differences in flow stress between unimplanted and implanted materials in indentation testing and but not in pillar testing, together with the differences in strain-burst behaviour and slip band morphology between unimplanted and implanted pillars, imply that the mechanisms giving rise to the measured flow stresses are different in nanoindentation and micropillar testing. In nanoindentation the maximum stress under the tip can reach several gigapascals. In unimplanted material this can give rise to the homogenous nucleation of dislocations as seen by the initial pop-ins[42], in the implanted conditions the stresses is sufficient to operate loops as dislocation sources, thus no initial pop-ins occur, this has also been seen by Armstrong et al. [6] in W5wt\%Ta. Due to the large density of loops acting as sources the effects of cross-slip around damage loops are likely to be invisible given the multiple operating and intersecting slip systems at the high strains in the indentation plastic zone. Thus while initial yield in the implanted material is easy there is then rapid hardening due to the dislocation loops in the damaged layer act as obstacles to flow giving rise to an increase in measured hardness. In micropillar testing the maximum stresses are much lower than indentation testing, as such the activation of dislocations controls the mechanical response rather than the propagation. In implanted micropillars the activation of dislocations still control the mechanical response but due to the increased number of obstacles and the ease of cross slip the observed deformation structure is very different.

The differences seen in yield properties between this study and the work on micropillars in proton irradiated copper by Kiener et al. [Error! Bookmark not defined.] are interesting. The defects in proton-irradiated copper are predominantly stacking fault tetrahedra (SFTs) with density $1 \times 10^{24} \mathrm{~m}^{-3}$ and diameter $0.5-2 \mathrm{~nm}$, a larger defect density compared to that seen in this and other ion implantation experiments. SFTs are not able to act as dislocation sources, and in copper the dissociation of screw dislocations will suppress cross slip as a mechanism to bypass the SFTs; the SFTs will thus act as strong obstacles to slip. These differences in radiation damage defect type and dislocation behaviour can account for the increase in yield stress seen 
between their unimplanted and implanted pillars of a similar diameter to those used in this study.

Finally the influence of the damage caused by the gallium ions should be considered. Work has shown that this damage can cause marked changes in measured mechanical properties between the same materials produced by FIB and by non-FIB routes [43]. In this study identical FIBbased manufacturing routes were used for both ion implanted and non-ion implanted pillars, which will be affected to the same degree by the gallium damage. How the gallium interacts with the iron ion-irradiation damage is unclear but will be limited to the top $30 \mathrm{~nm}$ of the pillars; the clear differences in behaviour between ion-irradiated and un-irradiated pillars show that the deep iron ion damage rather than shallow gallium ion damage is the controlling defect population.

\section{Conclusions}

In conclusion, the results reported here indicate that while there are marked differences between the deformation of micropillars in annealed and heavy-ion-irradiated material, the differences reflect the ease or difficulty of dislocation nucleation at relatively high stresses in these small test volumes, rather than differences in flow stresses translatable to testing of larger specimens. As such caution should be exercised when interpreting micropillar test data on irradiated materials in terms of bulk mechanical behaviour.

\section{Acknowledgements}

EG thanks the Worshipful Company of Founders, EPSRC and Rolls Royce Plc. for financial support. Implantations were carried out under EPSRC grants EP/F004451/1 and EP/H018921/1. DEJA thanks Culham Centre for Fusion Energy for support. All authors would like to thank Dr S. Fitzgerald (CCFE, UK) for useful discussions.

\section{Figure Captions}

Figure1: A) SEM micrograph of "sandwich sample" B) Hardness profile across one half of sandwich

Figure 2: SEM micrograph showing micropillar manufactured in ion damaged layer on the edge of the "sandwich sample"

Figure 3: Pure Fe: stress/strain curves from pillar compression.

Figure 4 A) Pure Fe unimplanted pillar before testing B). Pure Fe unimplanted pillar after testing to 500nm displacement. C) Pure Fe implanted pillar before testing. D) Pure Fe implanted pillar after testing to 500nm displacement.

Figure 5) TEM brightfield image in 111 zone with $g=110$, showing dislocation loops formed by ion implantation with an average diameter of $11 \mathrm{~nm}$ and a volume density of $4.5 \times 10^{21} \mathrm{~m}^{3}$. 


\section{References}

1, A. Kohyama, A. Hishinuma, Y. Kohno, K Shiba, A. Sagara, Fusion Eng and Des 41 (1998) 1

2, V. Barabash, G. Federici, M. Rödig, L.L. Snead, C.H. Wu, J Nuc Mater 283 (2000) 138-146

3 H. C. Schneider, B. Dafferner, J. Aktaa, J Nucl Mater Vol. 295, Issue 1(2001) pp16-20

4 C. Heintze, F. Bergner, M. Hernández-Mayoral, J Nuc Mater 417, (2011), 980

5 F.M. Halliday, D.E.J. Armstrong, J.D. Murphy, S.G. Roberts, Adv Mat Res 59 (2009) 304

6 D.E.J. Armstrong, A.J Wilkinson, S.G. Roberts, Phys Scripta, T145 (2011), 014076

7 P. Hosemann, C. Vieh, R.R. Greco, S. Kabra, J.A. Valdez,, M.J. Cappiello, S.A. Maloy J Nuc Mater 389 (2009) 239

8 K. Yabuuchia, H. Yanoa, R. Kasadab, H. Kishimotoc, A. Kimurab J Nuc Mater 417 (2011) 988

9 R. Kasada, Y. Takayama, K. Yabuuchi, A. Kimura, Fusion Eng Design, 86 (2011) 2658

10 P. Hosemann, D. Kiener, Y. Wang, S.A. Maloy, J Nuc Mater 425 (2012) 136

11 D.E.J. Armstrong, A.J. Wilkinson, S.G. Roberts, Phil Mag Let, 91, (2011) 394

12 D.E.J. Armstrong, A.J. Wikinson, S.G. Roberts, J. Mater. Res 24 (2009) 3268

13 A.T. Jennings and J. R. Greer, Phil Mag, 91, (2011) 1108

14 D.E.J. Armstrong.,A.J. Wilkinson, S.G. Roberts, Materials Research Society Symposium Proceedings 1185 (2009). 7

15 Michael D. Uchic, Dennis M. Dimiduk, Jeffrey N. Florando and William D. Nix, Science 305, (2004) 986-989

16, Michael D. Uchic, Paul A. Shade and Dennis M. Dimiduk, J Mater, 61(3) (2009)pp 36-41

17 Greer, J.R., De Hosson, J.T.M., Prog in Mater Scie 56 (6) (2011) pp. 654-724

18, Y. Yang, J.C. Ye, J. Lu, F.X. Liu, P.K. Liaw, Acta Mater, 57 (2009) 1613

19 D.M. Dimiduk, M.D. Uchic, T.A. Parthasarathy, Acta Mater 53 (2005) 4065

20, J.R. Greer, W.D. Nix, Phys Rev B, 73, (2006) 245410

21 Ng, K.S., Ngan, A.H.W Acta Mater 57 (16) ( 2009) 4902

22 M.A. Pouchon \& J. Chen \& R. Ghisleni \& J. Michler \& W. Hoffelner Experimental Mechanics 50 (2010) 79

23 P. Hosemann, Y. Dai, E. Stergar, H. Leitner, E. Olivas, A. T. Nelson and S. A. Maloy, Experimental Mechanics, 51 (2011) 1095

24. A.J. Bushby, S.G. Roberts, C.D. Hardie, J. Mater. Res. 27, (2012), 85.

25 G.F. Vander Voort Metallography principles and practice, (1999) New York: ASM International

26 D. Raabe, Phys. Stat. Sol. (a) 149, (1995) 575 
27 D Tabor The Hardness of Metals, OUP Oxford 2000

28 B.R. Lawn, R.F. Cook, J. Mater. Sci, 47 (2012) , 1.

29 M.D. Uchic, D.M. Dimiduk, Mat Sci Eng A, 400-401 (2005) 268-278

30 J.R. Greer, J-Y. Kim, M.J. Burek, J Mater, 61 (12) (2009)

31 J-Y Kim, J.R Greer, Acta Mat. 58 (2010) 2355

32 G. Monnet, D. Terentyev, Acta Mat. 57, (2009), 1416

33 C. Domain, G. Monnet, Phys. Rev. Let. 95 (2005) 215506

34 A. S. Schneider, D. Kaufmann, B. G. Clark, C. P. Frick, P. A. Gruber, R. Mönig, O. Kraft, and E. Arzt, Phys. Rev. let. 103 (2009) 105501

35 C.R. Weinberger, W. Cai, PNAS, 105 (2008) 14304

36 J.R. Greer, C.R. Weinberger, W. Cai, Mat. Sci. Eng. A. 493 (2008) 21

37 C.J. Healy, G J. Ackland, Mater. Res. Soc. Symp. Proc. Vol. 1369

38 R. Novokshanov and S.G. Roberts, University of Oxford UK, Unpublished data

39 P. A. Smidt Jr., Mastel, Phil. Mag, 20 (1969), 651

${ }^{40}$ Y. Chen, P. Spatig, M. Victoria J Nuc Mat 271\&272 (1999) 128

41 A. Arsenlis, M. Rhee, G. Hommes, R. Cook, J. Marian, Acta Mat, 60 (2012) 3748

42 Lorenz, A. Zeckzer, U. Hilpert, P. Grau, H. Johansen, and H. S. Leipner, PHYL REV B 67, (2003) 172101

43 S. Shim, H. Bei, M.K. Miller, G.M. Pharr, E.P. George, Acta. Mater.57 (2009) 503 

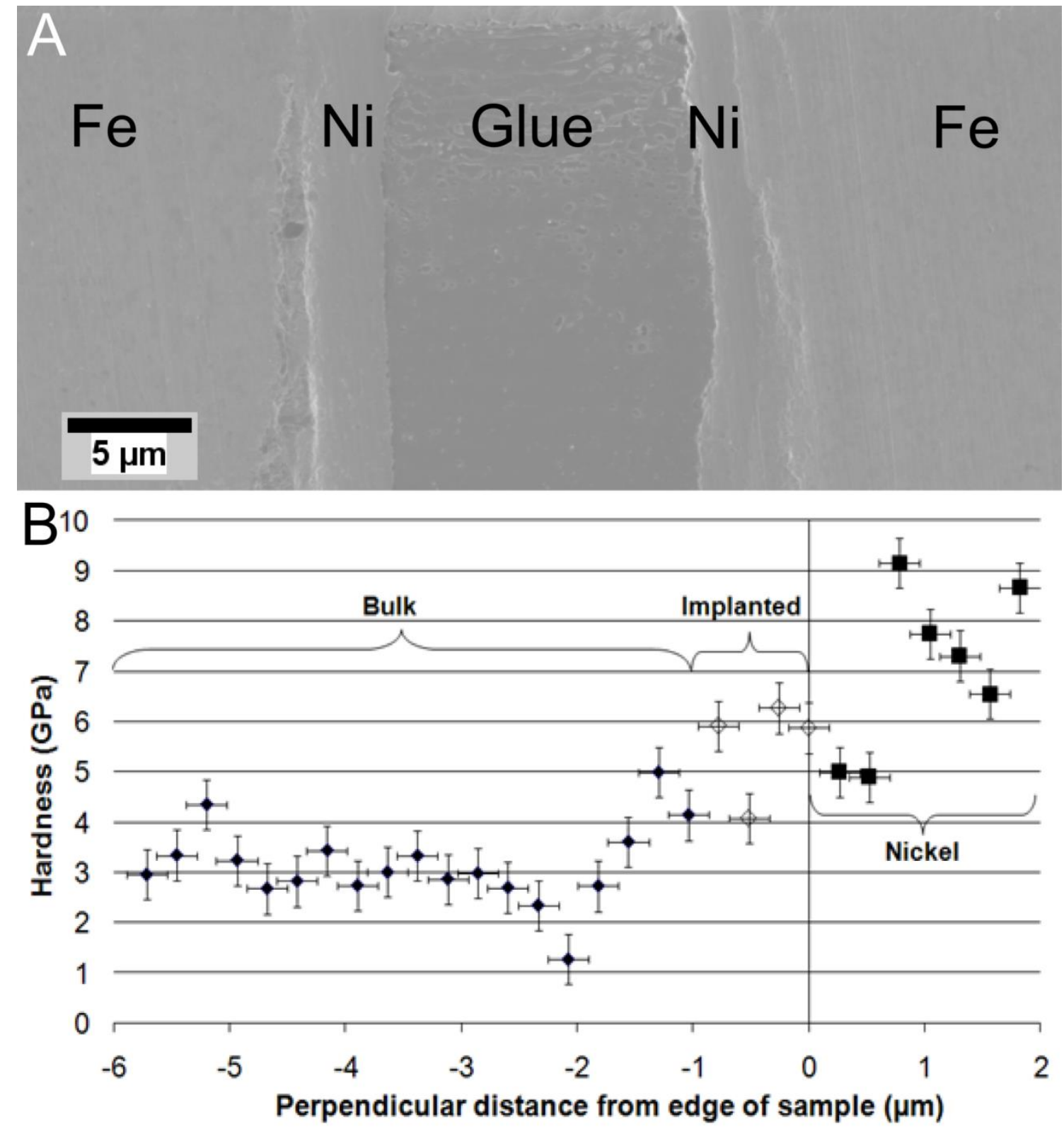

Figure1: A) SEM micrograph of "sandwich sample" B) Hardness profile across one half of sandwich 


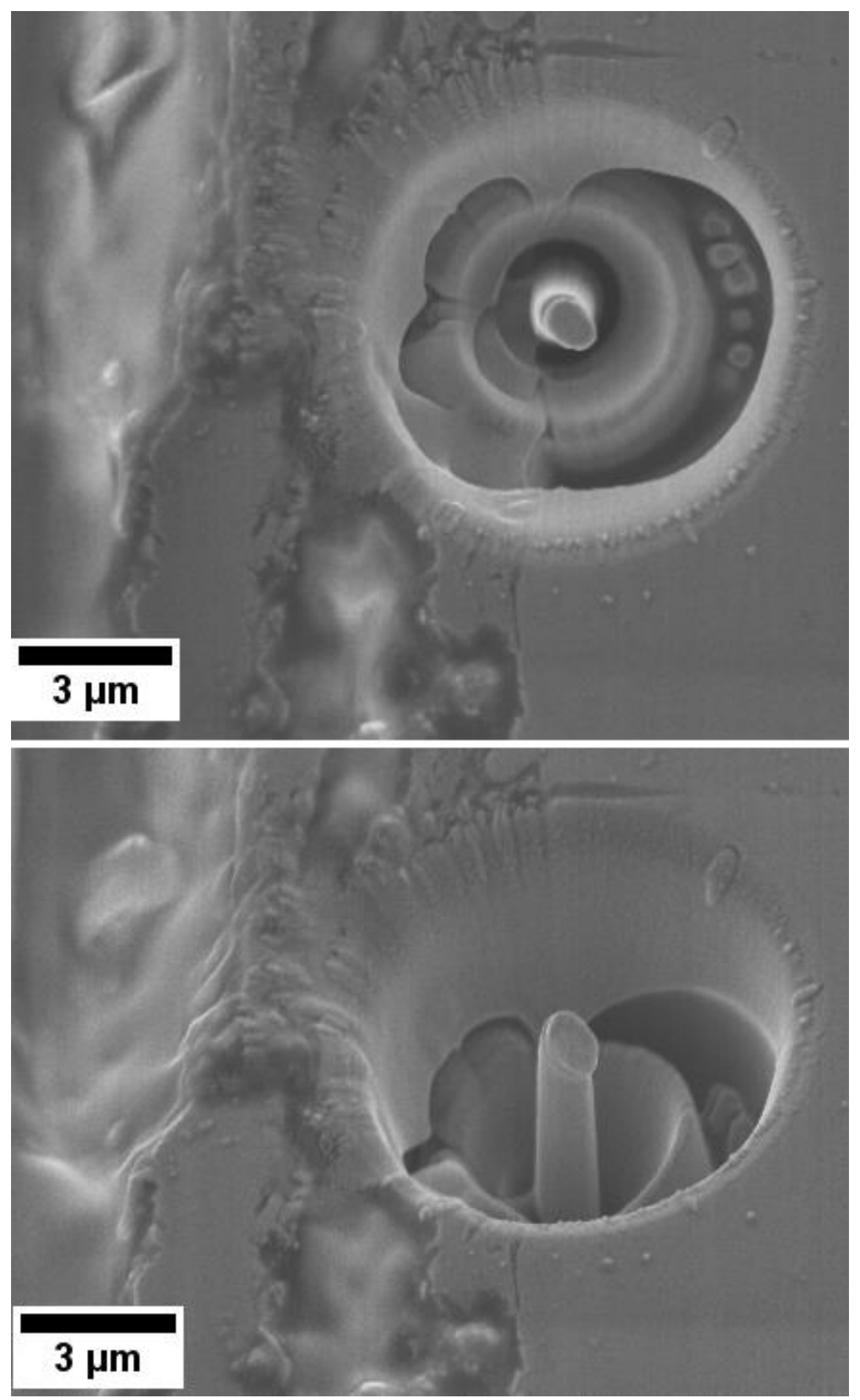

Figure 2: SEM micrograph showing micropillar manufactured in ion damaged layer on the edge of the "sandwich sample" 


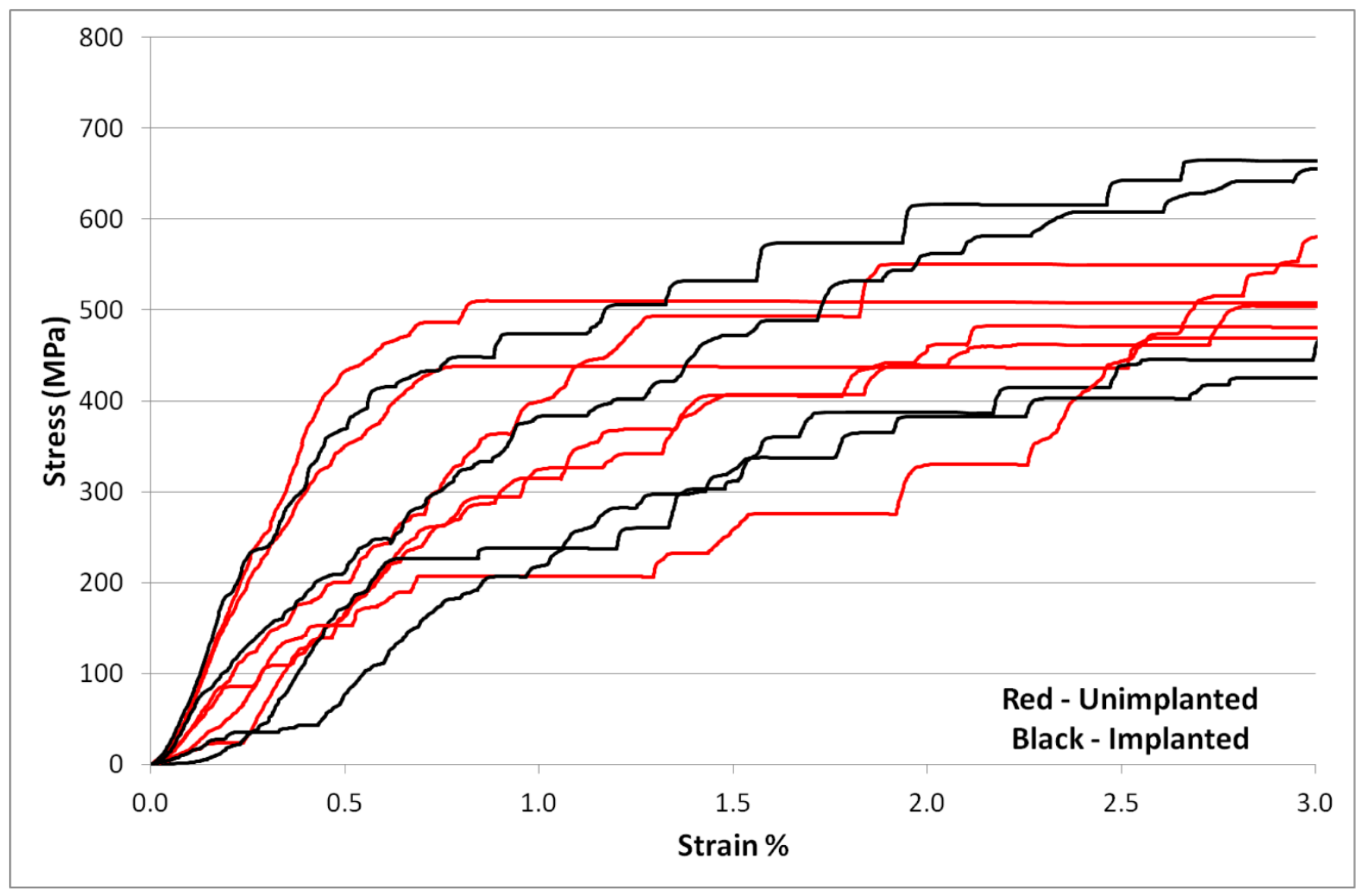

Figure 3: Pure Fe: stress/strain curves from pillar compression. 


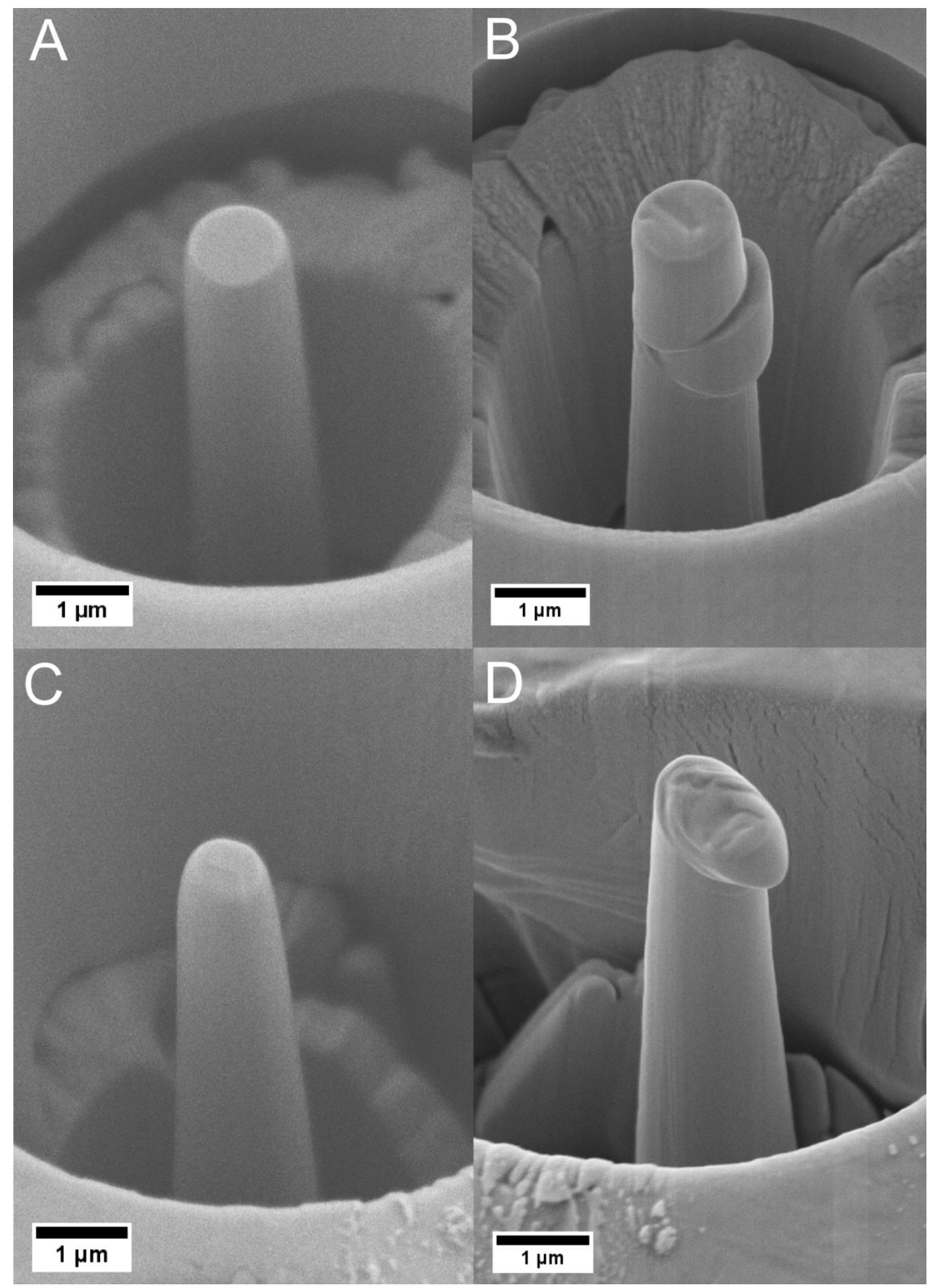

Figure 4 A) Pure Fe unimplanted pillar before testing B). Pure Fe unimplanted pillar after testing to $500 \mathrm{~nm}$ displacement.

C) Pure Fe implanted pillar before testing.

D) Pure Fe implanted pillar after testing to $500 \mathrm{~nm}$ displacement. 


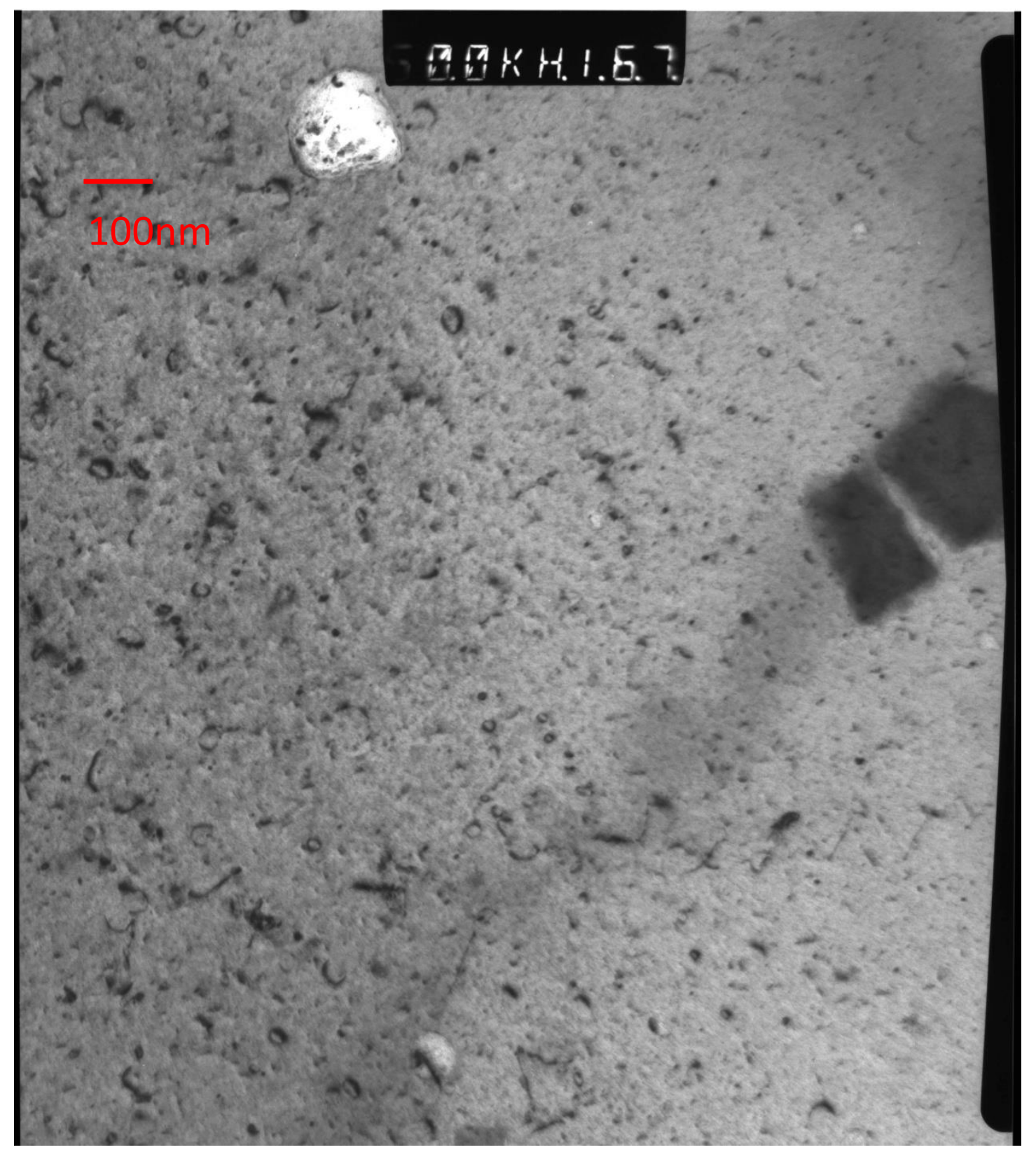

Figure 5) TEM brightfield image in 111 zone with $\mathrm{g}=110$, showing dislocation loops formed by ion implantation with an average diameter of $11 \mathrm{~nm}$ and a volume density of $4.5 \times 10^{21} \mathrm{~m}^{-3}$. 